\title{
Visual consensus feedback mechanism for group decision making with complementary linguistic preference relations
}

\author{
Francisco Chiclana ${ }^{1}, \mathrm{Jian}_{\mathrm{Wu}^{2}}$, and Enrique Herrera-Viedma ${ }^{3}$ \\ 1 Centre for Computational Intelligence, Faculty of Technology, De Montfort \\ University, Leicester, UK. chiclana@dmu.ac.uk \\ 2 School of Economics and Management, Zhejiang Normal University, Jinhua, \\ Zhejiang, China. jyajian@163.com \\ 3 Department of Computer Science and Artificial Intelligence, University of Granada, \\ Granada, Spain. viedma@decsai.ugr.es
}

\begin{abstract}
A visual consensus feedback mechanism for group decision making (GDM) problems with complementary linguistic preference relations is presented. Linguistic preferences are modelled using triangular fuzzy membership functions, and the concepts of similarity degree (SD) between two experts as well as the proximity degree (PD) between an expert and the rest of experts in the group are defined and used to measure the consensus level (CL). A feedback mechanism is proposed to identify experts, alternatives and corresponding preference values that contribute less to consensus. The novelty of this feedback mechanism is that it provides experts with visual representations of their consensus status to easily 'see' their consensus position within the group as well as to identify the alternatives and preference values that should be reconsidered for changing in the subsequent consensus round. The feedback mechanism also includes individualised recommendations to those identified experts on changing their identified preference values and visual graphical simulation of future consensus status if the recommended values were to be implemented.
\end{abstract}

Keywords: Group decisions making, Consensus, linguistic preferences, Visual feedback mechanism

\section{Introduction}

Subjectivity, imprecision and vagueness in the articulation of opinions pervade real world decision applications, and individuals usually find difficult to evaluate their preference using exact numbers. In these cases, individuals might feel more comfortable using words by means of linguistic labels or terms to articulate their preferences $[1,2]$.

Let $\mathcal{L}=\left\{l_{0}, \ldots, l_{s}\right\}$ be a set of linguistic labels $(s \geq 2)$, with semantics underlying a ranking relation that can be precisely captured with a linear order: $l_{0}<l_{1}<\cdots<l_{s}$. Assuming that the number of labels is odd and the central 
label $\left(l_{s / 2}\right)$ stands for the indifference state when comparing two alternatives, the remaining labels are usually located symmetrically around that central assessment, which guarantees that a kind of complementary or reciprocity property holds as in the case of numerical preferences [3]. Thus, if the linguistic assessment associated to the pair of alternatives $\left(x_{i}, x_{j}\right)$ is $r_{i j}=l_{h} \in \mathcal{L}$, then the linguistic assessment corresponding to the pair of alternatives $\left(x_{j}, x_{i}\right)$ would be $r_{j i}=l_{s-h}$. Therefore, the operator defined as $N\left(l_{h}\right)=l_{g}$ with $(g+h)=s$ is a negator operator because $N\left(N\left(l_{h}\right)\right)=N\left(l_{g}\right)=l_{h}[4]$.

The main two representation formats of linguistic information are [2]: the cardinal, which is based on the use of fuzzy sets characterised with membership functions and that are mathematically processed using Zadeh's extension principle [1]; and the ordinal, which is based on the use of the symbolic computational model [2]. Although the latter representation is able to capture some of the linguistic information to model, it is in fact processed using mathematical tools that are not appropriate for ordinal information but for information provided using a difference or ratio scale. Evidence of this is that the ordinal linguistic model is mathematically equivalent to the cardinal approach with fuzzy sets represented using a representative element of the corresponding membership functions, an example of which is the centroid [4]. Therefore, the uncertainty nature of the information is lost in the ordinal linguistic computational model. Furthermore, the linguistic cardinal approach is richer than the ordinal linguistic approach, not only because it has the latter one as a particular case but also because it provides a more flexible tool for GDM with LPRs because different types of fuzzy sets are possible to be used depending on the type and intensity of the imprecision and vagueness contained in the linguistic information to model.

In particular, convex normal fuzzy subsets of the real line, also known as fuzzy numbers, are commonly used to represent linguistic terms [5-7]. By doing this, each linguistic assessment is represented using a fuzzy number that is characterised by a membership function, with base variable the unit interval $[0,1]$, describing its semantic meaning. The membership function maps each value in $[0,1]$ to a degree of performance representing its compatibility with the linguistic assessment [1]. This paper focuses on the use of triangular fuzzy numbers to model linguistic information, which leads to the so-called triangular fuzzy complementary preference relations (TFCPRs) [8] because they extend both numeric preference relations and interval-valued preference relations.

GDM problems generally involve situations of conflict among its experts, and therefore it is preferable that the set of experts reach consensus before applying a selection process to derive the decision solution. There are two basic consensus models in GDM: the static consensus models [9] and the interactive consensus models [10]. The former does not implement any type of feedback mechanism to advice experts on how to change their preferences in order to achieve a higher consensus level while the latter does. Existing interactive consensus models methodology relies on the imposition to decision makers (DM) of changes in their opinion when consensus is below a threshold value. However, in practice, it is up to the decision maker to implement or not the recommen- 
dations given to him/her [11]. A more reasonable and suitable policy should rest on this premise and, consequently, it would allow the DM to revisit his/her evaluations using appropriate and meaningful consensus information representation. Therefore, the aim of this paper is to propose a visual consensus feedback mechanism for GDM to provide experts with visual representations of their consensus status to easily 'see' their consensus position within the group as well as to identify the alternatives and preference values that he/she should reconsider for changing in the subsequent consensus round. The feedback mechanism also includes individualised recommendations to those identified experts on changing their identified preference values as well as visual graphical simulation of future consensus status if the recommended values were to be implemented. To achieve this, we first define a TFCPRs similarity degree (SD) to measure, in the unit interval, how close two individual experts are. The proximity of an expert with respect to the whole group of experts is also measured, resulting in individual proximity degree (PD). Consensus level (CL) is defined as a linear combination of SD with PD, and all will be defined at the three different levels of a preference relation [12-14]: the pairs of alternatives, the alternatives and the whole set of alternatives.

The rest of paper is set out as follows: Section 2 focuses on the development of similarity and proximity degrees for TFCPRs. In Section 3, the level of consensus for TFCPRs is proposed, and a visual information feedback mechanism to increase the level of consensus is investigated. Finally, conclusions are drawn in Section 4.

\section{Similarity and proximity degrees of triangular fuzzy complementary preference relations}

A fuzzy subset $\widetilde{A}$ of $\mathbb{R}$ is called a triangular fuzzy number (TFN) when its membership function $\mu_{\widetilde{A}}(x): \mathbb{R} \rightarrow[0,1]$ is $[15]$ :

$$
\mu_{\widetilde{A}}(x)= \begin{cases}0, & x<a \\ \frac{x-a}{b-a}, & a<x \leq b \\ \frac{c-x}{c-b}, & b \leq x<c \\ 0, & x>c\end{cases}
$$

A TFN is shortly represented as $\widetilde{A}=(a, b, c)$, with $a$ and $c$ known as the lower and upper bounds, respectively, while $b$ is known as its modal value.

A preference relation on a set of alternatives $X=\left\{x_{1}, x_{2}, \ldots, x_{n}\right\}$ with elements being TFNs, $\widetilde{P}=\left(\widetilde{p}_{i j}\right)_{n \times n}$ and $\widetilde{p}_{i j}=\left(a_{i j}, b_{i j}, c_{i j}\right)$, is called a triangular fuzzy complementary preference relation (TFCPR) if the following property holds [15]:

$$
a_{i j}+c_{j i}=b_{i j}+b_{j i}=c_{i j}+a_{j i}=1, \forall i, j=1,2, \ldots n,
$$




\subsection{Similarity degrees}

Given two TFNs, $\widetilde{A}_{1}=\left(a_{1}, b_{1}, c_{1}\right)$ and $\widetilde{A}_{2}=\left(a_{2}, b_{2}, c_{2}\right)$, their similarity $d\left(\widetilde{A}_{1}, \widetilde{A}_{2}\right)$ can be defined as follows $[16,12]$ :

$$
s\left(\widetilde{A}_{1}, \widetilde{A}_{2}\right)=1-\frac{\left|a_{1}-a_{2}\right|+\left|b_{1}-b_{2}\right|+\left|c_{1}-c_{2}\right|}{3} .
$$

In the following, the similarity degree between two experts using TFCPRs is introduced:

Definition 1 Let $P^{h}=\left(p_{i k}^{h}\right)$ and $P^{l}=\left(p_{i k}^{l}\right)$ be two TFCPRs on a set of alternatives $X$ provided by two experts $e_{h}$ and $e_{l}$, respectively. Then, the similarity degree between experts $e_{h}$ and $e_{l}$ on the pair of alternatives $\left(x_{i}, x_{k}\right), S D_{i k}^{h l}$, is :

$$
S D_{i k}^{h l}=S D\left(p_{i k}^{h}, p_{i k}^{l}\right)=1-d\left(p_{i k}^{h}, p_{i k}^{l}\right) .
$$

Notice that $S D_{i k}^{h l}=1$ implies $\left|a_{i k}^{h}-a_{i k}^{l}\right|=\left|b_{i k}^{h}-b_{i k}^{l}\right|=\left|c_{i k}^{h}-c_{i k}^{l}\right|=0$ and therefore $p_{i k}^{h}=p_{i k}^{l}$. Therefore, we have the following interpretation: the higher the value of $S D_{i k}^{h l}$, the more similar $p_{i k}^{h}$ and $p_{i k}^{l}$ are.

Definition 2 The similarity degree between experts $e_{h}$ and $e_{l}$ on the alternative $x_{i}$ is: $S D_{i}^{h l}=S D\left(p_{i}^{h}, p_{i}^{l}\right)=\frac{\sum_{k=1}^{n} S D\left(p_{i k}^{h}, p_{i k}^{l}\right)}{n}$.

As above, when $S D_{i}^{h l}=1$ experts $e_{h}$ and $e_{l}$ provide the same linguistic valuations for pairs of alternatives involving $x_{i}$. Thus, the higher the value of $S D_{i}^{h l}$, the more similar the experts' preferences are on the alternative $x_{i}$.

Definition 3 The similarity degree between experts $e_{h}$ and $e_{l}$ on the whole set of alternatives $X$ is: $S D^{h l}=S D\left(P^{h}, P^{l}\right)=\frac{\sum_{i=1}^{n} \sum_{k=1}^{n} S D\left(p_{i k}^{h}, p_{i k}^{l}\right)}{n^{2}}$.

Clearly, $S D^{h l}=1$ means that experts $e_{h}$ and $e_{l}$ provide identical TFCPRs, and we can interpret this similarity degree as follows: the higher the value $S D^{h l}$, the closer experts $e_{h}$ and $e_{l}$ are in their preferences on the set of alternatives.

The similarity degrees of an expert with the rest of the group of experts at the three different levels of a relation are defined as:

Level 1. Similarity degree on the pair of alternatives $\left(x_{i}, x_{k}\right)$ of expert $e_{h}$ to the rest of experts in the group is $S P A_{i k}^{h}=\frac{\sum_{l=1, l \neq h}^{m} S D_{i k}^{h l}}{m-1}$.

Level 2. Similarity degree on the alternative $x_{i}$ of expert $e_{h}$ to the rest of experts in the group is $S A_{i}^{h}=\frac{\sum_{k=1}^{n} S P A_{i k}^{h}}{n}$.

Level 3. Similarity degree on the preference relation of expert $e_{h}$ to the rest of experts in the group is $S D^{h}=\frac{\sum_{i=1}^{n} S A_{i}^{h}}{n}$. 
Finally, each expert in the GDM problem can be associated a relative (normalised) importance degree based on the similarity degrees at level 3 computed above, which we obviously refer to as the relative similarity degree of an expert: $R S D^{h}=\frac{S D^{h}}{\sum_{l=1}^{m} S D^{t}}$. These relative importance degrees could be different to particular importance weights the experts in the group are assigned before they provide their linguistic information on the set of alternatives. Our methodology is to implement both importance degrees in the computation of consensus to reflect the actual position of experts in the group as a collective $[17,18]$. This will be developed in the following subsection. Next we provide a simple GDM example to illustrate the computation of the similarity degrees at the three levels of a relation and the final relative similarity degrees of the experts in the group.

Example 1. Suppose four experts $\left\{e_{1}, e_{2}, e_{3}, e_{4}\right\}$ with associated importance degrees $I D=(0.2,0.1,0.4,0.3)^{T}$, are asked to provide their preference on a set of four alternatives $\left\{x_{1}, x_{2}, x_{3}, x_{4}\right\}$, being their linguistic preferences modelled via the following TFCPRs:

$$
\begin{aligned}
P^{\mathbf{1}}= & \left(\begin{array}{cccc}
- & (0.3,0.4,0.5) & (0.4,0.5,0.6) & (0.5,0.6,0.7) \\
(0.5,0.6,0.7) & - & (0.4,0.5,0.6) & (0.3,0.4,0.5) \\
(0.4,0.5,0.6) & (0.4,0.5,0.6) & - & (0.5,0.6,0.7) \\
(0.3,0.4,0.5) & (0.5,0.6,0.7) & (0.3,0.4,0.5) & -
\end{array}\right) \\
P^{\mathbf{2}}= & \left(\begin{array}{cccc}
- & (0.4,0.5,0.6) & (0.2,0.3,0.4) & (0.3,0.4,0.5) \\
(0.4,0.5,0.6) & - & (0.5,0.6,0.7) & (0.5,0.6,0.7) \\
(0.6,0.7,0.8) & (0.3,0.4,0.5) & - & (0.1,0.2,0.3) \\
(0.5,0.6,0.7) & (0.3,0.4,0.5) & (0.7,0.8,0.9) & -
\end{array}\right) \\
P^{\mathbf{3}}= & \left(\begin{array}{cccc}
- & (0.5,0.6,0.7) & (0.4,0.5,0.6) & (0.6,0.7,0.8) \\
(0.3,0.4,0.5) & - & (0.5,0.6,0.7) & (0.2,0.3,0.4) \\
(0.4,0.5,0.6) & (0.3,0.4,0.5) & - & (0.4,0.5,0.6) \\
(0.2,0.3,0.4) & (0.6,0.7,0.8) & (0.4,0.5,0.6) & -
\end{array}\right) \\
P^{\mathbf{4}}= & \left(\begin{array}{cccc}
- & (0.4,0.5,0.6) & (0.5,0.6,0.7) & (0.5,0.6,0.7) \\
(0.4,0.5,0.6) & - & (0.6,0.7,0.8) & (0.2,0.3,0.4) \\
(0.3,0.4,0.5) & (0.2,0.3,0.4) & - & (0.3,0.4,0.5) \\
(0.3,0.4,0.5) & (0.6,0.7,0.8) & (0.5,0.6,0.7) & -
\end{array}\right)
\end{aligned}
$$

I) The similarity degree on pairs of alternatives for each expert are:

$$
\begin{aligned}
& S P A^{1}=\left(\begin{array}{llll}
1.000 & 0.867 & 0.900 & 0.900 \\
0.867 & 1.000 & 0.867 & 0.867 \\
0.900 & 0.867 & 1.000 & 0.767 \\
0.900 & 0.867 & 0.767 & 1.000
\end{array}\right) ; S P A^{2}=\left(\begin{array}{llll}
1.000 & 0.933 & 0.767 & 0.767 \\
0.933 & 1.000 & 0.933 & 0.733 \\
0.767 & 0.933 & 1.000 & 0.700 \\
0.767 & 0.733 & 0.700 & 1.000
\end{array}\right) \\
& S P A^{3}=\left(\begin{array}{llll}
1.000 & 0.867 & 0.900 & 0.833 \\
0.867 & 1.000 & 0.933 & 0.867 \\
0.900 & 0.933 & 1.000 & 0.833 \\
0.833 & 0.867 & 0.833 & 1.000
\end{array}\right) ; S P A^{4}=\left(\begin{array}{llll}
1.000 & 0.933 & 0.833 & 0.900 \\
0.933 & 1.000 & 0.867 & 0.867 \\
0.833 & 0.867 & 1.000 & 0.833 \\
0.900 & 0.867 & 0.833 & 1.000
\end{array}\right)
\end{aligned}
$$


II) The similarity degrees on alternatives for each expert are:

$$
\begin{aligned}
& S A^{1}=(0.917,0.900,0.883,0.883) ; S A^{2}=(0.867,0.900,0.850,0.800) \\
& S A^{3}=(0.900,0.917,0.917,0.883) ; S A^{4}=(0.917,0.917,0.883,0.900)
\end{aligned}
$$

III) The similarity degrees on the set of alternatives for each expert are:

$$
S D^{1}=0.896 ; S D^{2}=0.854 ; S D^{3}=0.904 ; S D^{4}=0.904 .
$$

IV) The relative group similarity degrees for each expert are:

$$
R S D^{1}=0.252 ; R S D^{2}=0.240 ; R S D^{3}=0.254 ; R S D^{4}=0.254 .
$$

\section{$2.2 \quad$ Proximity degrees}

The proximity degrees measure the similarity between individual experts' opinions and the collective opinion for the group of experts. The aggregation of individual opinions will be weighted using a weight vector whose elements are a linear combination of the importance degree of individuals before the decision making process and the relative similarity degrees computed based on the information they provided as per the previous subsection. This is elaborated next:

(1) Experts weighting vector: $W=\eta \cdot I D+(1-\eta) \cdot R S D$. If $\eta>0.5$, then the group/moderator values higher the a priori importance degrees of the experts than their a posteriori relative similarity degrees. Obviously, for homogeneous GDM problems the value $\eta=0$ applies.

(2) The collective TFCPR, $P=\left(p_{i k}\right)_{n \times n}$, is computed as follows:

$$
p_{i k}=w^{1} \otimes p_{i k}^{1} \oplus w^{2} \otimes p_{i k}^{2} \oplus \cdots \oplus w^{m} \otimes p_{i k}^{m}
$$

Example 2 (Example 1 Continuation). Assuming a value of $\eta=0.5$ we have the following weighting vector

$$
W=0.5 * I D+0.5 * R S D=(0.22,0.17,0.33,0.28)^{T}
$$

and the collective TFCPR is

$$
P=\left(\begin{array}{cccc}
- & (0.41,0.51,0.61) & (0.39,0.49,0.59) & (0.50,0.60,0.70) \\
(0.39,0.49,0.59) & - & (0.51,0.61,0.71) & (0.27,0.37,0.47) \\
(0.41,0.51,0.61) & (0.29,0.39,0.49) & - & (0.34,0.44,0.54) \\
(0.30,0.40,0.50) & (0.53,0.63,0.73) & (0.46,0.56,0.66) & -
\end{array}\right)
$$

Once the collective TFCPR is obtained, we compute the proximity degrees for each expert at the three different levels of a relation:

Level 1. Proximity degree on pair of alternatives $\left(x_{i}, x_{k}\right)$ of expert $e_{h}$ to the group is $P P A_{i k}^{h}=S D\left(p_{i k}^{h}, p_{i k}\right)$. 
Level 2. Proximity degree on alternatives $x_{i}$ of expert $e_{h}$ to the group is $P A_{i}^{h}=$ $\underline{\sum_{k=1}^{n} P P A_{i k}^{h}}$.

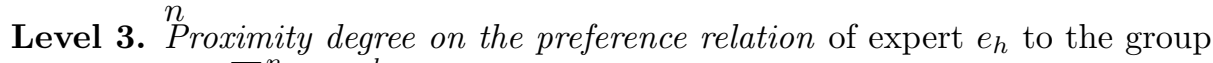
is $P D^{h}=\frac{\sum_{i=1}^{n} P A_{i}^{h}}{n}$.

Example 3 (Example 1 Continuation). Proximity degrees computation.

I) The proximity degree on pairs of alternatives for each expert are:

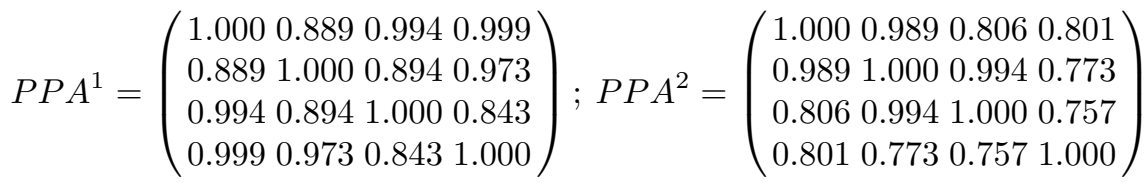

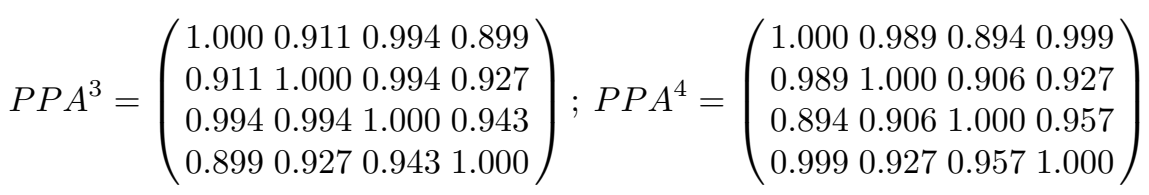

II) The proximity degrees on alternatives for each expert are:

$$
\begin{aligned}
& P A^{1}=(0.971,0.939,0.933,0.954) ; P A^{2}=(0.899,0.939,0.889,0.833) \\
& P A^{3}=(0.951,0.958,0.983,0.942) ; P A^{4}=(0.971,0.956,0.940,0.971)
\end{aligned}
$$

III) The proximity degrees on the relation for each expert are:

$$
P D^{1}=0.949 ; P D^{2}=0.890 ; P D^{3}=0.958 ; P D^{4}=0.959 .
$$

\section{Consensus model with visual information feedback mechanism for GDM with TFCPRs}

Both similarity degree (SD) and proximity degree (PD) convey the concept of closeness of opinions between experts in a group: the first one between pairs of individual experts and the second one between an individual expert and the rest of experts in the group. Thus, both degrees could/should be used in measuring the level of consensus within a group of experts regarding the set of feasible alternatives in GDM. The simplest of the combinations is the linear one, and it is here used to propose the following definitions of the consensus level (CL) associated to each expert of the group at the three different levels of a relation:

Level 1. Consensus level on the pair of alternatives (CLPA) $\left(x_{i}, x_{k}\right)$ of expert $e_{h}$ is $C L P A_{i k}^{h}=\psi \cdot S P A_{i k}^{h}+(1-\psi) \cdot P P A_{i k}^{h}$.

Level 2. Consensus level on the alternatives $(C L A) x_{i}$ of expert $e_{h}$ is $C L A_{i}^{h}=$ $\psi \cdot S A_{i}^{h}+(1-\psi) \cdot P A_{i}^{h}$. 
Level 3. Consensus level on the relation (CL) of expert $e_{h}$ is $C L^{h}=\psi \cdot S D^{h}+$ $(1-\psi) \cdot P D^{h}$.

The parameter $\psi \in[0,1]$ controls the weight of both similarity and proximity criteria. Unless there are specific reasons to prefer one index to the other one, the value to assume for the weighting parameter $\psi$ should be 0.5 , as it is assumed in the example below.

Example 4 (Example 1 Continuation). Consensus levels computation. Setting $\psi$ at 0.5 , the following consensus levels on the relation are obtained:

$$
C L^{1}=0.922, C L^{2}=0.872, C L^{3}=0.932, C L^{4}=0.932
$$

The only expert with a consensus level below the threshold value is $e_{2}$ and therefore he/she will receive feedback advice on how to change his/her preferences to achieve a higher consensus level.

In practice, it is rare to achieve full and unanimous agreement of all the experts regarding all the feasible alternatives. As a consequence, the consensus threshold value $(\gamma)$ to achieve is usually set to a value lower than 1 . At the same time, the decision output should be acceptable for at least half of the experts, which means that the parameter $\gamma$ should be set to a value no lower than 0.5 . If the consensus level is not acceptable, that is, if it is lower than the specified threshold value, the experts are normally invited to discuss their opinions further in an effort to make them closer. To help experts in their discussion, in the following section a detailed description of a visual feedback methodology is provided.

\subsection{Visual Information Feedback Mechanism}

The visual information feedback mechanism consists of three stages: firstly, the identification of the triangular fuzzy preference values that should be subject to modification; secondly, the generation of advice on the direction-value of the required change; and, thirdly, the automatic feedback process simulation to show what would happen if experts are to accept the recommended preference values. These three stages are described in detail below:

(1) Identification of the Triangular Fuzzy Preference Values: The set of triangular fuzzy preference values that contribute less to reach an acceptable consensus level is identified and presented to the experts using visual graphs as illustrated in Fig. 1. Once consensus levels are computed, at the relation level, all experts will receive a visual representation of their consensus status in relation to the threshold value, which can be used to easily identify the experts furthest form the group. Following with Ex. 4, and using a threshold value $\gamma=0.9$, Fig. 1(a) clearly identifies expert $e_{2}$ as the only expert contributing less to group consensus. If necessary, individual visual representations of consensus levels of alternatives and pair of alternatives are also 
provided to each expert to help them identify those alternatives and their associated preference values at the level of pairs of alternatives that contribute less to consensus and, consequently, potential to be reconsidered for changing in the next round of consensus. It is obvious from Ex. 4 that this is necessary to be done for expert $e_{2}$, whom would receive visual representation at these levels as illustrated in Fig. 1(b) and Fig. 1(c), respectively.

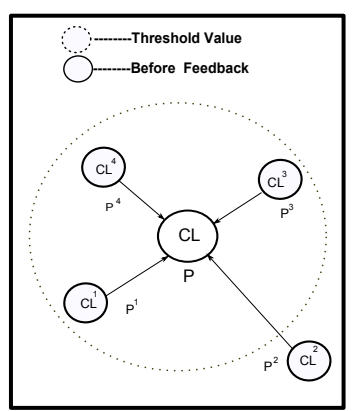

(a) Consensus levels on the relation: $C L^{h}$

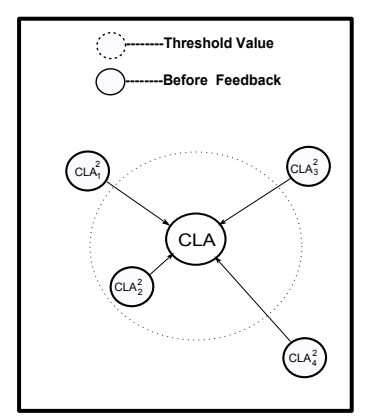

(b) Consensus levels on the alternatives for $e_{2}: C L A_{i}^{2}$

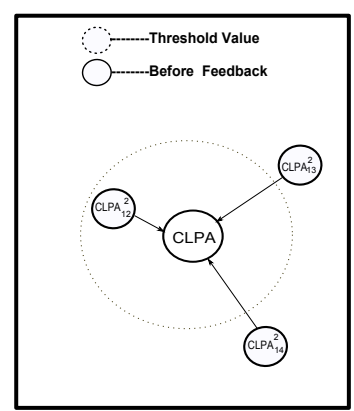

(c) Consensus levels on the pairs of alternatives for $A_{1}$ and $e_{2}: C L P A_{1 j}^{2}$

Fig. 1. Visual representation of consensus levels in relation to the consensus threshold value

Mathematically, these steps are modelled as follows:

Step 1. The set of experts with consensus levels below the threshold value $\gamma$ is identified: $E C H=\left\{h \mid C L^{h}<\gamma\right\}$.

Step 2. For experts identified in step 1 , those alternatives with a consensus level below $\gamma$ are identified: $A C H=\left\{(h, i) \mid h \in E C H \wedge C L A_{i}^{h}<\gamma\right\}$.

Step 3. Finally, the triangular fuzzy preference values for the experts and alternatives identified in steps 1 and 2 that need to be changed are identified: $P A C H=\left\{(h, i, k) \mid(h, i) \in A C H \wedge C L P A_{i k}^{h}<\gamma\right\}$.

Example 5 (Example 1 Continuation). The sets of 3-tuple identified as contributing less to consensus are:

$P A C H=\{(2,1,3),(2,1,4),(2,2,4),(2,3,1),(2,3,4),(2,4,1),(2,4,2),(2,4,3)\}$

(2) Generation of Advice: The feedback mechanism also generates personalised recommendations rules, which will not only tell the experts which preference values they should change, but also provide them with the consensus advice to revisit their evaluation in the light of this extra information. For all $(h, i, k) \in P A C H$, the following rule is feed backed to the corresponding experts: 
"To increase your consensus level (CL), your preference value $p_{i k}^{h}$ should be closer to $\overline{\bar{p}}_{i k}^{h}=\psi \cdot \bar{p}_{i k}^{h}+(1-\psi) \cdot p_{i k}, "$ where $\bar{p}_{i k}^{h}=\left(\sum_{l=1, l \neq h}^{m} p_{i k}^{l}\right) /(m-1)$ and $p_{i k}$ the collective preference value. The reciprocity property that the TFCPRs verify implies that when the pair of alternatives $(i, k)$ is identified for change, the pair $(k, i)$ has to be changed accordingly as well.

Example 6 ((Example 1 continuation)). The recommendations for expert $e_{2}$ are:

- Preference value $p_{13}^{2}$ should be closer to $(0.4,0.5,0.6)$.

- Preference value $p_{31}^{2}$ should be closer to $(0.4,0.5,0.6)$.

- Preference value $p_{14}^{2}$ should be closer to $(0.5,0.6,0.7)$.

- Preference value $p_{41}^{2}$ should be closer to $(0.3,0.4,0.5)$.

- Preference value $p_{24}^{2}$ should be closer to $(0.4,0.5,0.6)$.

- Preference value $p_{42}^{2}$ should be closer to $(0.4,0.5,0.6)$.

- Preference value $p_{34}^{2}$ should be closer to $(0.4,0.5,0.6)$.

- Preference value $p_{43}^{2}$ should be closer to $(0.4,0.5,0.6)$.

(3) Automatic Feedback Process Simulation: A what-if scenario analysis could be run to generate a visual graphical simulation of future consensus status if the recommended values were to be implemented, as shown in Fig. 2(a), Fig. 2(b) and Fig. 2(c). This will provide the decision makers with a clear picture of their actual position within the group, which they can then use to decide upon their actual position or subsequent action. If the advice is implemented, then the consensus level increases as example 7 shows. Not implementing these advices can lead to the consensus level to remain fixed or to increase at a very low rate, which would make the group consensus threshold value difficult to achieve. To avoid these situation, a maximum number of iterations maxIter can be incorporated in the visual information feedback mechanism following a similar approach of consensus models proposed in [19].

Example 7 (Finishing Example 1). After expert $e_{2}$ revisits his/her evaluation and implements the recommended TFNs, a new round of the consensus process is carried out, leading to the following new consensus levels:

$$
C L^{1}=0.956, C L^{2}=0.976, C L^{3}=0.961, C L^{4}=0.957 .
$$

Because all experts are over the minimum consensus threshold value $\gamma=0.9$, the consensual collective TFCPR is computed from which the final solution of consensus will be selected.

\section{Conclusion}

In this paper, a novel visual information feedback mechanism for GDM problems with TFCPRs has been presented. To achieve this, the concepts of similarity degree (SD) between two experts as well as the proximity degree (PD) between an 

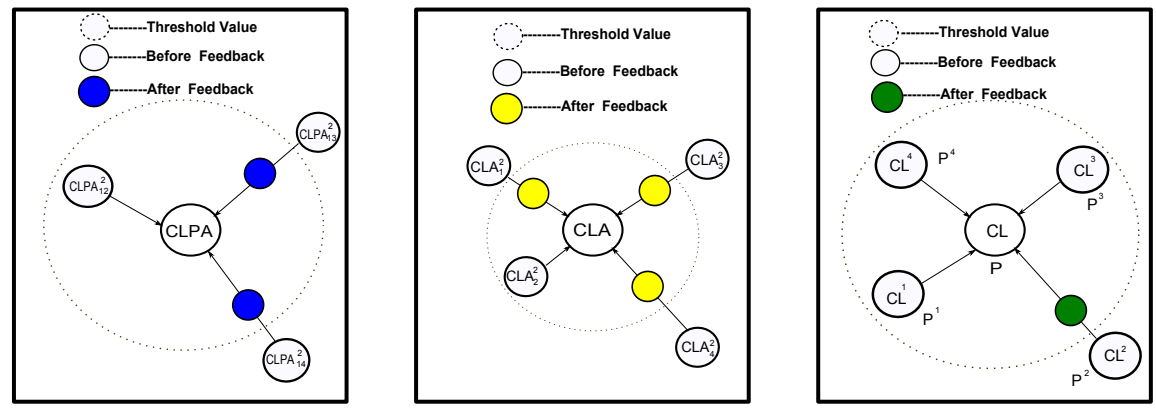

(a) $C L P A_{1 j}^{2}$ before and af- (b) $C L A_{i}^{2}$ before and af- (c) $C L^{h}$ before and after $e_{2}$ implements recom- ter $e_{2}$ implements recom- ter $e_{2}$ implements recommended values mended values

mended values

Fig. 2. Simulation of consensus before and after recommended values are implemented by expert $e_{2}$

experts and the rest of experts in the group are developed for TFCPRs. These degrees are used to compute both the aggregation weighting vector as well as the consensus level of the group of experts. The visual information feedback mechanism is investigated to identify experts, alternatives and corresponding preference values that contribute less to consensus. Recommendations to help experts the direction of the change required to increase their consensus are produced and an automatic visual feedback process simulation to show the experts what would happen if they were to follow recommendations by pictures is developed.

\section{Acknowledgements}

The authors would like to acknowledge FEDER financial support from the Project FUZZYLING-II Project TIN2010-17876; the financial support from the Andalusian Excellence Projects TIC-05299 and TIC-5991; and the University of Granada Excellence campus GENIL-BioTIC-UGR Research Visit programme. This work was also supported by National Natural Science Foundation of China (NSFC) under the Grant No.71101131 and No.713311002, and Zhejiang Provincial National Science Foundation for Distinguished Young Scholars of China under the Grant No. LR13G010001.

\section{References}

1. Zadeh, L.A. The concept of a linguistic variable and its application to approximate reasoning-I. Information Sciences, 8, 199 - 249, 1975.

2. Herrera F., Alonso S., Chiclana F., Herrera-Viedma E. Computing with words in decision making: foundations, trends and prospects. Fuzzy Optimization and Decision Making 8 (4), 337 - 364, 2009. 
3. Chiclana, F, Herrera-Viedma, E., Alonso, S., Herrera, F. Cardinal consistency of reciprocal preference relations: a characterization of multiplicative transitivity. IEEE Transactions on Fuzzy Systems, 17 (1), 14 - 23, 2009.

4. Pérez-Asurmendi, P., Chiclana, F. Linguistic majorities with difference in support. Applied Soft Computing 18, 196 - 208, 2014.

5. Zhou, S-M., Chiclana, F., John, R., Garibaldi, J. M. Type-1 OWA operators for aggregating uncertain information with uncertain weights induced by type-2 linguistic quantifiers. Fuzzy Sets and Systems 159 (24), 3281 - 3296, 2008.

6. Zhou, S-M. , Chiclana, F., John, R., Garibaldi, J. M.. Alpha-level aggregation: a practical approach to type-1 OWA operation for aggregating uncertain information with applications to breast cancer treatments. IEEE Transactions on Knowledge and Data Engineering 23 (10), 1455 -1468, 2011.

7. Chiclana, F., Zhou, S-M. Type-reduction of general type-2 fuzzy sets: the type-1 OWA approach. International Journal of Intelligent Systems 28 (5), 505-522, 2013.

8. Xia, M. M., Xu, Z. S. Methods for fuzzy complementary preference relations based on multiplicative consistency. Computers and Industrial Engineering 61 (2011) 930-935.

9. Xu, Z. S., Cai, X. Q. Group consensus algorithms based on preference relations. Information Science 181 (2011) 150-162.

10. Alonso, S., Herrera-Viedma, E., Chiclana, F., Herrera, F. A web based consensus support system for group decision making problems and incomplete preferences. Information Sciences 180 (2010) 4477-4495.

11. Eklund, P., Rusinowska,A., de Swart, H., Dong, Y. C., Xu, Y. F., Li, H., Feng, B. A consensus model of political decision-making. Annals of Operations Research 158 (2008) 5-20.

12. Chiclana, F., Tapia-Garcia,J. M., del Moral, M. J., Herrera-Viedma, E. A statistical comparative study of different similarity measures of consensus in group decision making. Information Sciences 221 (2013) 110-123.

13. Alonso, S., Pérez, I.J., Cabrerizo, F.J., Herrera-Viedma, E.. A linguistic consensus model for Web 2.0 communities. Applied Soft Computing 13 (1) (2013) 149-157.

14. Wu, J., Chiclana, F. A social network analysis trust-consensus based approach to group decision-making problems with interval-valued fuzzy reciprocal preference relations. Knowledge-Based Systems 59 (2014) 97-107.

15. Laarhoven, P. J. M., Pedrycz, W. A fuzzy extension of Saaty's priority theory. Fuzzy Sets Systems 11 (1983) 229-241.

16. Zwick, R., Carlstein, E., Budescu, D. V. Measures of similarity among fuzzy concepts: A comparative analysis. International Journal of Approximate Reasoning 1 (1987) 221-242.

17. Chiclana, F., Herrera-Viedma, E., Herrera, F., Alonso, S. Some induced ordered weighted averaging operators and their use for solving group decision-making problems based on fuzzy preference relations. European Journal of Operational Research 182 (2007) 383-399.

18. Mata, F., Perez, L.G., Zhou, S.-M., Chiclana, F. Type-1 OWA methodology to consensus reaching processes in multi-granular linguistic contexts. KnowledgeBased Systems 58 (2014) 11-22.

19. Herrera-Viedma, E., Herrera, F., Chiclana, F. A consensus model for multiperson decision making with different preference structures. IEEE Transactions on Systems, Man, and Cybernetics - Part A: Systems and Humans 32 (2002) 394-402. 